\title{
Effects and Alternatives to Haematinics on Anemic Patients-A Questionnaire Study
}

\author{
Dharsan.R ${ }^{1}$, Brundha M.P ${ }^{* 2}$ and Lesile Rani ${ }^{3}$ \\ ${ }^{1}$ Undergraduate Student, Department of General Pathology, Saveetha Dental College, Saveetha \\ Institute of Medical and Technical Sciences, Chennai-600077, Tamil Nadu, India. \\ ${ }^{2}$ Associate professor, Department of General Pathology, Saveetha Dental College, Saveetha \\ Institute of Medical and Technical Sciences, Chennai-600077, Tamil Nadu, India. \\ ${ }^{3}$ Department of General Pathology, Saveetha Dental College, Saveetha Institute of Medical and \\ Technical Sciences, 162, Poonamallee High Road, Chennai-600077, Tamil Nadu, India.
}

\section{ABSTRACT}

Study of the alternatives and side effects of haematinics on anaemic patients. Anaemia is a condition which can be either hereditary or acquired with an abnormal RBC. Anemia are usually treated by Haematinics in the form of tablets, capsules and syrup and sometimes injection. Long term intake of haematinics produces some side effects like Gastritis, tooth staining, etc. This study was conducted to analyse the side effects experienced by anemia patients due to haematinics. This is a survey based study, A questionnaire was prepared regarding hematinics and alternate to haematinics. From the derived data the difficulty and side effects of haematinics were studied and analysed.Adverse effects of oral iron: epigastric pain, nausea, vomiting, gastritis, metallic taste, constipation (due to astringent effect) or diarrhea (irritant effect) are the usual adverse effects. Liquid preparations of iron causes staining of teeth. Many people preferred natural alternatives to haematinics. The survey says that some groups of people preferred natural products over hematinic drugs for their treatment of anaemia due to its side effects.

KEY WORDS: ANAEMIA, HAEMATINICS, HEMATOPOIESIS , IRON , VITAMIN B12, FOLIC ACID..

\section{INTRODUCTION}

Anaemia is a condition which can be either hereditary or acquired with an abnormal RBC. (Guralnik et al., 2004) The major disorders observed commonly in the pro facial disorders are namely- anaemia, lymphoid disorder, bone marrow and immune deficiency and HIV virus. (Kilgore, 2006)Anaemia is common nowadays and it is caused due to decreased levels of red blood cells in an individual.

\section{ARTICLE INFORMATION}

*Corresponding Author: brundha.sdc@gmail.com

Received 3rd August 2020 Accepted after revision 23rd Sep 2020

Print ISSN: 0974-6455 Online ISSN: 2321-4007 CODEN: BBRCBA

Thomson Reuters ISI Web of Science Clarivate Analytics USA and Crossref Indexed Journal

\section{Clarivate
Analytics}

NAAS Journal Score 2020 (4.31) SJIF: 2020 (7.728)

A Society of Science and Nature Publication,

Bhopal India 2020. All rights reserved.

Online Contents Available at: http//www.bbrc.in/

Doi: $h t t p: / / d x$.doi.org/10.21786/bbrc/13.8/109
Hematinics- nutrient required for hematopoiesis. The main hematinics are iron, vitamin B12 and folate. Deficiency in these leads to anaemia. (Muretto, Angelucci and Lucarelli, 2002).

Iron plays an important role in certain basic physiological processes, particularly the synthesis of haemoglobin and myoglobin. Dietary sources rich in iron are meat, eggs and leafy vegetables. Increased absorption occurs when iron stores are diminished, and when erythropoiesis is active.(Klipstein-Grobusch et al., 1999) For adult males, $1 \mathrm{mg}$ of iron absorbed each day is probably sufficient, but for adult females, $2 \mathrm{mg}$ per day are required, and for pregnant females, 3 to $3.5 \mathrm{mg}$ per day are necessary to meet the increased demands. Iron deficiency is a common disease in modern society, and is likely to remain so. It is not a definitive diagnosis, but a symptom complex, and in every instance where iron deficiency is demonstrated, a cause must be sought and identified with confidence

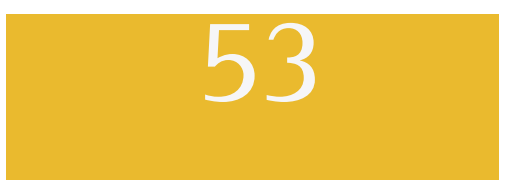


before treatment is commenced. An adequate history and physical examination are important in reaching this diagnosis; aided by a full blood picture, and, in most instances, a determination of the serum iron level and latent iron binding capacity. A careful menstrual history in iron-deficient women is essential.(Jiang et al., 2004)

Vitamin B12 is synthesised in higher animals indirectly from bacterial sources, and for man to have an adequate vitamin B12 intake, some form of dietary meat and animal products is essential. Hydroxocobalamin is a more efficient therapeutic agent than cyanocobalamin, and should be given by injection.(Montgomery, 1995) The daily requirement for vitamin B12 for the healthy adult is between 2 and $5 \mu \mathrm{g}$, representing up to $70 \%$ of the dietary vitamin. Smaller doses will produce a therapeutic response in deficient states, but will not sustain health. The metabolism of vitamin B12 within the body is complex, and is not as clearly understood as the body's handling of iron. In the investigation of a patient with megaloblastic anaemia, the nature, extent and cause of the underlying disorder must be assessed. Peripheral blood cell counts and examination of cell morphology in the peripheral blood and bone marrow will assist the diagnosis.

Vitamin B12 deficiency may be diagnosed when low vitamin B12 serum levels have been demonstrated, when methylmalonic acid excretion in the urine is low, and when an optimum response to a standard dose of vitamin B12 is achieved. When vitamin B12 deficiency has been diagnosed, gastric causes should be sought in the first instance, usually by gastroscopy, testing for histaminefast achlorhydria, and by demonstrating the failure to absorb a radioactive tracer dose of vitamin B12, which is absorbed when the defect is corrected by the addition of intrinsic factor.(de Sousa and Porto, 1998) If a gastric cause cannot be implicated, an intestinal cause of failure of absorption is likely. Dietary inadequacy leading to vitamin B12 deficiency is unusual, except in persons who are entirely vegetarian.

When a megaloblastic anaemia is demonstrated in the presence of pregnancy, during anticonvulsant therapy for epilepsy, or in the chronic alcoholic, folate deficiency is commonly the underlying cause. However, folate deficiency may be due to inadequate dietary intake, intestinal malabsorption, or an increased requirement due to cellular turnover, and in a number of cases, more than one of these factors may be involved.(Herbert et al., 1994) The diagnosis of folate deficiency requires the exclusion of vitamin B12 deficiency, demonstration of low serum folate, and possibly, a low red cell folate. Here again, the haematological response to a therapeutic dose of folate (200 $\mu \mathrm{g}$ daily, preferably by injection) is an important diagnostic feature. (Smith et al., 1997).

The body's folates are obtained from plant and animal foodstuffs, particularly liver and yeast, and they exist as polyglutamates of tetrahydrofolate. The dietary requirement (free folate) is between 150 and $200 \mu \mathrm{g}$ per day. (Valk, de Valk and Marx, 1999). This study is conducted to assess and evaluate the effects caused by haematinics taken for longer duration by anaemia patients. And also to create awareness on the natural food and vegetables rich in iron and has the ability to increase RBC cells and Haemoglobin levels. (Nelson, 2009)

\section{MATERIAL AND METHODS}

This is a survey based study. A questionnaire was prepared regarding hematinics and anaemia .Anaemic patients were asked to fill the survey. It was attended by 100 patients. The questionnaire initially consisted of participant's demographic data such as the Age and gender The later questions were about their treatment, duration of treatment and side effects by haematinics. Once the required responses were received, the survey was closed and the data was taken and analysed statistically.

\section{RESULTS AND DISCUSSION}

The treatment of choice for iron deficiency is iron, and it can and should be given orally in sufficient quantities to restore the body's storage to normal levels, in addition to achieve the desired elevation of the haemoglobin level. As a therapeutic agent for anemia,iron is simple,cheap and it is significantly effective in treating anemia . The simplest and most useful form of iron is ferrous sulphate, taken orally. Iron is one of the few therapeutic agents where oral therapy is more predictable and more effective than the systematically administered form.(Valk, de Valk and Marx, 1999) Many oral iron preparations exist, but they are no more efficient in producing an optimal haemoglobin response than ferrous sulphate. There may, however, be a need in some instances to use preparations other than ferrous sulphate in an endeavour to minimise symptoms of gastro-intestinal intolerance, an aspect which has been unduly emphasised in the past.(Valk, de Valk and Marx, 1999; Goddard et al., 2011).

Figure 1: Age group with Anaemia: From the 100 anaemic patients who had taken up the survey $9 \%$ (9 patients) regardless of sex were below 16 years of age, 34\% were between the age of 16 to 24,around $25 \%$ of them were between 24 years to 30 years of age and 32\% of patients were above the age of 30 .

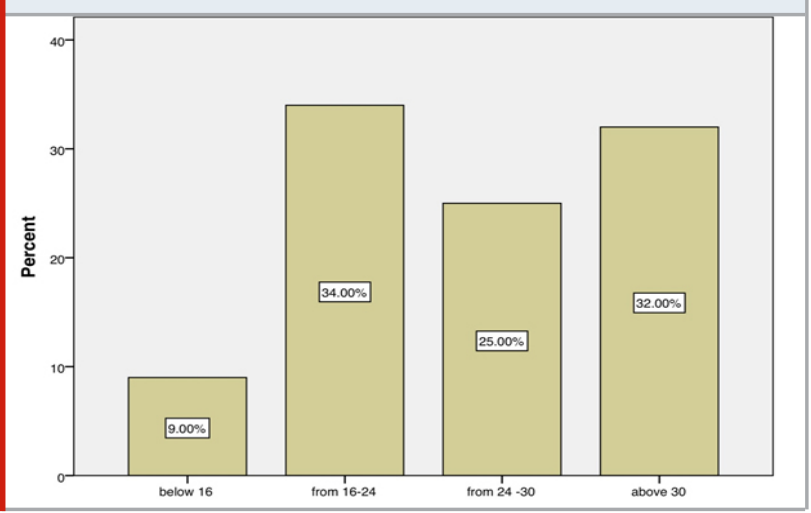


The principal benefit of slow or sustained release preparations appears to be the convenience of once-daily administration and their palatability; they do not appear to be any more efficient in delivering the desired amounts of iron to the body.(Mukhopadhyay and Mohanaruban, 2002) Intravenous and intramuscular iron are indicated rarely, in such instances as profound iron deficiency in a pregnant patient (to increase iron stores rapidly), where self-medication is unreliable, where there is genuine and severe gastro-intestinal intolerance, or where there is a clearly demonstrable gastro-intestinal absorptive defect. (Murphy and Hutchinson, 1994) Iron poisoning is due to the indigestion of iron, mainly in children where a fatal dose could be as little as $1 \mathrm{~g}$ of ferrous sulphate. The treatment of iron poisoning is now well defined and includes use of the chelating agent desferrioxamine to assist in the removal of excessive amounts of the ingested iron.

Figure 2: Forms of Drug Intake which explains about the various forms of haematinic intake. Among 100 respondents, $66 \%$ of patients where under taking tablets(Folic acid,Iron,Vit B complex), where 20\% were taking haematinics in form of syrups(iron), $2 \%$ of the patients were taking parenteral forms of iron and Vit B12 through injections.

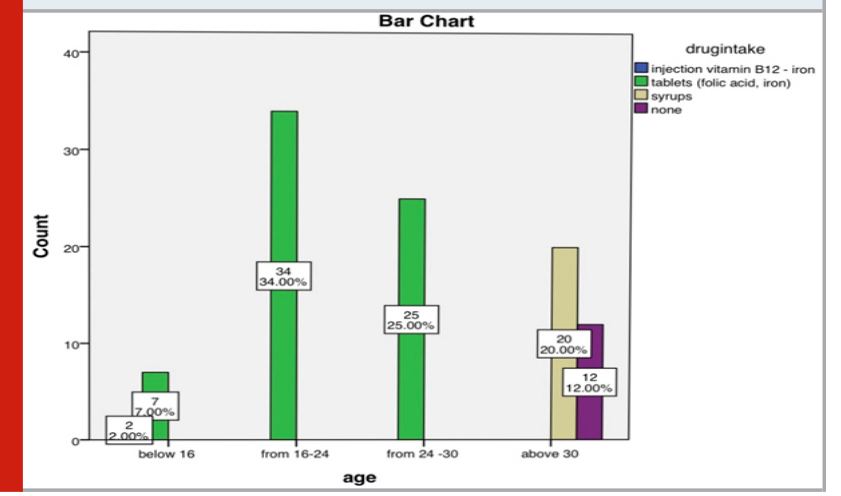

(Guyatt et al., 1992; Murphy and Hutchinson, 1994) The aim is to achieve a haematological remission and to replace the body stores of vitamin B12.(Smith, 2000) Oral vitamin B12 is indicated only in the patient in whom injections are undesirable (e.g. bleeding disorder). If the response to vitamin B12 therapy is poor or absent, it is likely that the megaloblastic anaemia is due to folate deficiency.(Patterson, 2003)

Oral administration of $5 \mathrm{mg}$ folic acid is more than adequate in treating a deficiency state, even in the patient with intestinal malabsorption. Long-term folate therapy is probably required only in older people who have a nutritional deficiency which is not likely to be remedied by improvement in their dietetic habits.(Rimon et al., 2005) Prophylactic folate is probably desirable in pregnancy, given together with an iron supplement. Because the blood volume is usually normal or increased in megaloblastic anaemia, blood transfusion is seldom if ever indicated, except in the most unusual circumstances. (Daugherty, 2008)
Adverse effects of Iron: Adverse effects of oral iron: epigastric pain, nausea, vomiting, gastritis, metallic taste, constipation (due to astringent effect) or diarrhea (irritant effect) are the usual adverse effects.(Wolf et al., 2020) Liquid preparations of iron causes staining of teeth. (Högberg and Lindvall, 1964)

Figure 3: Bar chart explains the Treatment of the anaemic patient. $21 \%$ of patients were under haematinics, $8 \%$ of them never used haematinics, whereas $71 \%$ of those patients gave up haematinics as they attained normal levels of haemoglobin and some were affected by the side effects.

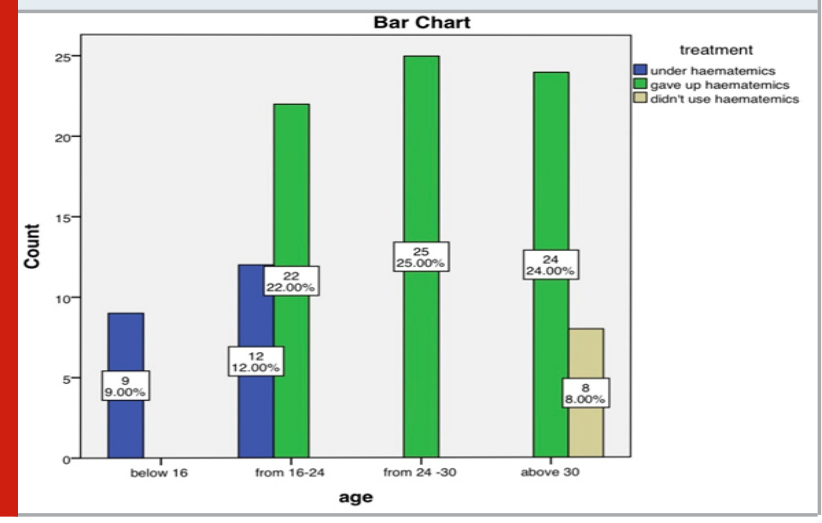

Figure 4: Shows the patients reasons for giving up Haematinics, where $18 \%$ of the patients were still under haematinics, $11 \%$ of them had given up as their RBC level has reached adequate, whereas $40 \%$ had shifted to natural alternatives and the rest $40 \%$ of the patients had given up on haematinics due to its adverse effects

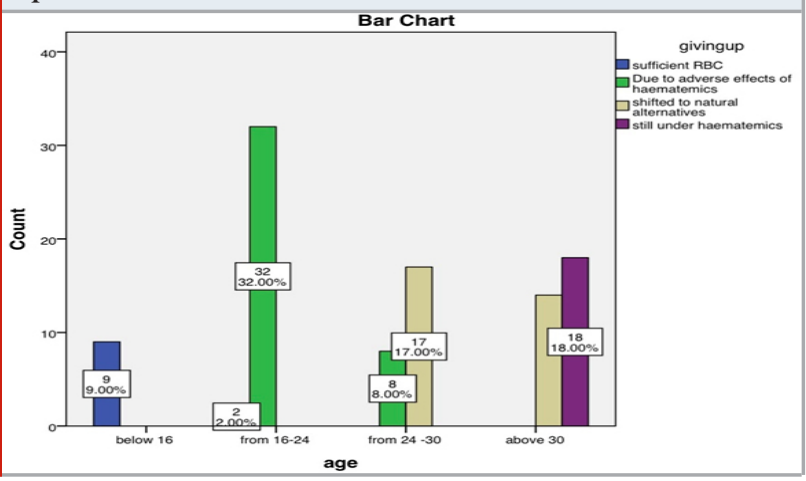

Adverse effects on parenteral iron: Local- Pain at the site of injection, pigmentation of the skin and sterile abscess are seen. Systemic-fever, headache, joint pain, palpitation, difficulty in breathing, lymph node enlargement, and rarely anaphylaxis.(Tripathi, 2013a) Acute iron poisoning is common in infants and children in the womb, if the pregnant mother consumes about 10 tablets(1-2g). Manifestations include vomiting, abdominal pain, hematemesis, bloody diarrhea, shock, drowsiness, cyanosis, acidosis, dehydration, (Tripathi, 2013b)cardiovascular collapse and coma. Immediate diagnosis and treatment are important as death may occur in 6-12 hours.(Vani and Grover, 2003) 
Natural foods that level iron deficiency - For anemia: There are many types of anemia. The most common type is iron deficiency anemia. Red blood cells contain a protein called hemoglobin. Hemoglobin is full of iron. Without sufficient iron, your body can't make the hemoglobin it needs to create enough red blood cells to deliver oxygen-rich blood throughout your body. (Tripathi, 2008)

A lack of folate and vitamin B-12 may also impact your body's ability to make red blood cells. If your body can't process B-12 properly, you may develop pernicious anemia. Natural food diet rich in iron and vitamin B helps in treating anemia.(Letsky, 1983)

Figure 5: Shows that $26 \%$ of those who undertook haematinics complained of its teeth staining and bad breath, 23\% complained of gastritis due to haematinics, $7 \%$ said they had heartburn, 11\% complained of ulcer, $17 \%$ said they had constipation due to haematinics, $11 \%$ complained of nausea and vomiting and 5\% complained of diarrhoea.

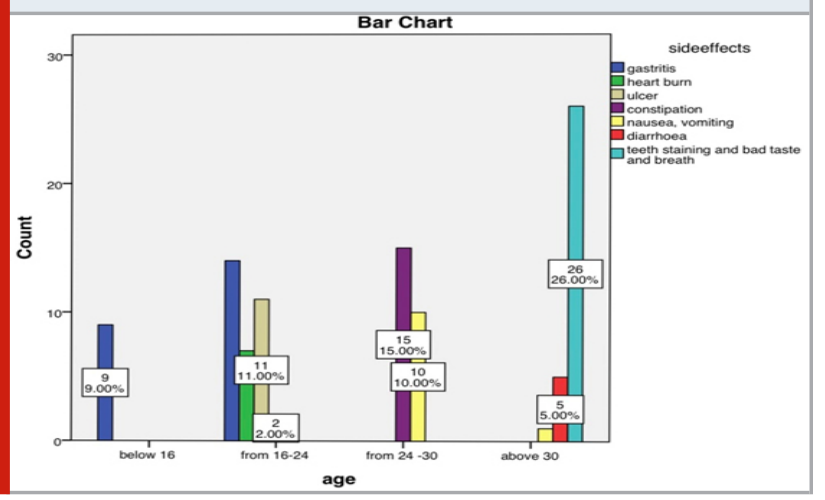

Figure 6: 26\% of those used leafy greens as an alternative to haematinics, $23 \%$ took meat, poultry for their treatment, $4 \%$ consumed liver, 13\% consumed seafood, 16\% consumed beans and 18\% consumed nuts and seeds and alternatives to haematinics so that it would help them with their anaemia.

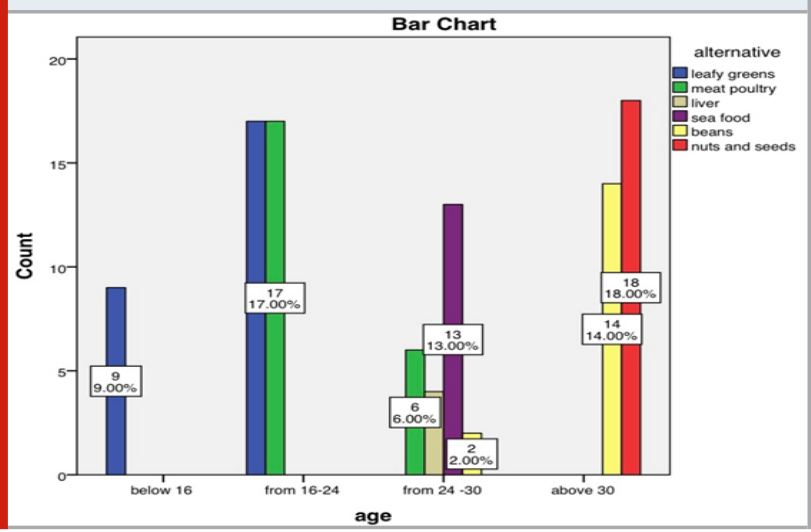

1. Leafy green vegetables: Leafy greens, especially dark ones, are among the best sources of nonheme iron. They include:spinach,kale,collard greens, dandelion greens,Swiss chard. There are also folate-like leafy greens such as Swiss chard and collard greens. Citrus fruits, beans, and whole grains are good sources of folate. Some green leafy vegetables are high in iron, such as spinach and kale, are also high in oxalates. Oxalates are compounds stopping nonheme iron absorption. So, though consuming your greens as part of an overall diet of anemia is helpful, do not rely solely on them to treat your disease.(Burz et al., 2019) Vitamin C helps your stomach absorb iron.Leafy greens can increase iron absorption by eating food containing vitamin C, including oranges, red peppers \&t strawberries. Some greens, such as collard greens and Swiss chard, are healthy sources of both iron and vitamin C.

2. Meat and poultry: All meat and poultry contain heme iron. The best examples are red meat, lamb and poison. Poultry and chicken have lower amounts. Meat or poultry can increase iron absorption from nonheme foods, such as leafy greens.

3. Liver: Most people are scared about organ meats, but they are a great source of iron. Liver is arguably the most popular organ meat. It's rich in iron and folate. Some other iron-rich organ meats are heart, kidney, and beef tongue.(Abdel-Razeq and Hashem, 2020)

4. Seafood: Some seafood provides heme iron. Shellfish such as oysters, clams, and shrimp are good sources. Most fish contain iron. Fish high in iron include:sardines, canned in oil,canned or fresh tun,fresh salmon,fresh halibut,fresh perch, fresh haddock.

While both fresh and canned salmon are good sources of iron, the amount of calcium found in canned salmon is small. Calcium binds with iron and reduces its absorption. Calcium-high foods should not be eaten with iron-rich foods at the same time.

5. Fortified foods: Many foods are fortified with iron. Vegetarians who struggle to est other sources of iron could effectively make use of fortified foods. They include-fortified orange juice,fortified, ready-to-eat cereals,foods made from fortified refined flour such as white bread,fortified pasta,foods made from fortified cornmeal,fortified white rice.

6. Beans: Beans are good sources of iron for vegetarians and meat eaters alike. They're also inexpensive and versatile. Some iron-rich options are:kidney beans, chickpeas, soybeans, black-eyed peas, pinto beans,black beans,peas,lima beans

7. Nuts and seeds: Many types of nuts and seeds are good sources of iron. They taste great on their own or sprinkled on salads or yogurt.it is wise to choose raw nuts.. Some nuts and seeds that contain iron are:pumpkin seeds, cashews,pistachios, hemp seeds, pine nuts,sunflower seeds Almonds are also a good source of iron. They are perfect for a balanced diet, but since they are high in calcium, they can not increase the level of iron too much. (Pietra, Degrassi and Fusi-Schmidhauser, 2020) 
Takeaway: No one food will cure anemia. However a balanced diet full of dark, leafy greens, noodles and seeds, fish, meat and beans, will help you to get the anemia you need. A skillet made of cast iron is a standard anemia diet. Foods cooked in cast iron absorb iron from the skillet.The most iron consumed by acidic foods and the least soluble foods cooked during short periods. Recall these recommendations if you adopt a diet plan for anemia:

Don't consume iron-rich foods that obstruct iron absorption with food or beverages. These include coffee or tea, eggs, foods high in oxalates, and foods high in calcium. To increase absorption, eat foods rich in iron that are high in vitamin C, such as bananas, tomatoes or strawberries. To boost absorption eat iron-rich foods with beta-carotene-containing foods, such as apricots, red peppers and beets. Eat a variety of heme and nonheme iron foods all day to increase your intake of iron. Use both heme and nonheme iron foods to improve iron absorption whenever possible. Add foods rich in folate and vitamin B-12 to support red blood cell production. (Golberg and Martin, 1964)

\section{CONCLUSION}

Hence by the survey we can conclude that people preferred natural products over hematinic drugs for their treatment of anaemia due its side effects. Majority of the study population prefer daily intake of natural products like green leafy vegetables, nuts, fruits helps in prevention and also in treating anaemia. The study population aged 16 years and 24 years were mostly affected by anemia, they were advised to take hematinics and majority of them gave up hematinics after 2-3 months of treatment due to its adverse effects.

\section{ACKNOWLEDGEMENTS}

The authors are thankful to Saveetha Dental college for providing a platform for carrying out the research.

Conflict Of Interest: The authors declare no conflict of interest.

\section{REFERENCES}

Abdel-Razeq, H. and Hashem, H. (2020) 'Recent update in the pathogenesis and treatment of chemotherapy and cancer induced anemia', Critical reviews in oncology/ hematology, 145, p. 102837.

Burz, C. et al. (2019) 'Iron-Deficiency Anemia', Iron Deficiency Anemia. doi: 10.5772/intechopen.80940.

Daugherty, K. K. (2008) '2008 Current Medical Diagnosis and Treatment, 47th Edition2008 Current Medical Diagnosis and Treatment, 47th Edition Edited by McPheeStephen J MD, PapadakisMaxine A MD, and TierneyLawrence MJr MD. Published by McGraw-Hill, New York, NY, 2008. ISBN 978-0-07149430-4. Paperbound, xviii 1673 pp. $(25.5 \times 18.5$ cm), \$71.95.www.mcgraw-hill medical.com', Annals of Pharmacotherapy, pp. 1523-1523. doi: 10.1345/ aph. 11280.

Goddard, A. F. et al. (2011) 'Guidelines for the management of iron deficiency anaemia', Gut, pp. 1309-1316. doi: 10.1136/gut.2010.228874.

Golberg, L. and Martin, L. E. (1964) 'Iron-containing Haematinics', Evaluation of Drug Activities, pp. 535583. doi: 10.1016/b978-1-4832-2846-4.50008-7.

Guralnik, J. M. et al. (2004) 'Prevalence of anemia in persons 65 years and older in the United States: evidence for a high rate of unexplained anemia', Blood, 104(8), pp. 2263-2268.

Guyatt, G. H. et al. (1992) 'Laboratory diagnosis of irondeficiency anemia: an overview', Journal of general internal medicine, 7(2), pp. 145-153.

Herbert, V. et al. (1994) 'Most free-radical injury is iron-related: it is promoted by iron, hemin, holoferritin and vitamin $\mathrm{C}$, and inhibited by desferoxamine and apoferritin', Stem cells, 12(3), pp. 289-303.

Högberg, K.-G. and Lindvall, S. (1964) 'the Distribution Of Parenteral Iron Haematinics In Nonpregnant, Pregnant And Lactating Rats', British Journal of Pharmacology and Chemotherapy, pp. 275-288. doi: 10.1111/j.1476-5381.1964.tb02033.x.

Jiang, R. et al. (2004) 'Body iron stores in relation to risk of type 2 diabetes in apparently healthy women', JAMA: the journal of the American Medical Association, 291(6), pp. 711-717.

Kilgore, C. (2006) 'Supplements of Benefit to Only Some Elderly’, Family Practice News, p. 64. doi: 10.1016/ s0300-7073(06)73053-5.

Klipstein-Grobusch, K. et al. (1999) 'Serum ferritin and risk of myocardial infarction in the elderly: the Rotterdam Study', The American journal of clinical nutrition, 69(6), pp. 1231-1236.

Letsky, E. (1983) 'Anaemia and haematinics in pregnancy', Clinical Pharmacology in Obstetrics, pp. 28-48. doi: 10.1016/b978-0-7236-0652-9.50009-9.

Montgomery, E. B., Jr (1995) 'Heavy metals and the etiology of Parkinson's disease and other movement disorders', Toxicology, 97(1-3), pp. 3-9.

Mukhopadhyay, D. and Mohanaruban, K. (2002) 'Iron deficiency anaemia in older people: investigation, management and treatment', Age and ageing, 31(2), pp. 87-91.

Muretto, P., Angelucci, E. and Lucarelli, G. (2002) 'Reversibility of cirrhosis in patients cured of thalassemia by bone marrow transplantation', Annals of internal medicine, 136(9), pp. 667-672.

Murphy, P. T. and Hutchinson, R. M. (1994) 'Identification and treatment of anaemia in older patients', Drugs \& aging, 4(2), pp. 113-127.

Nelson, R. L. (2009) 'Iron and Colorectal Cancer Risk: Human Studies', Nutrition Reviews, pp. 140-148. doi: 10.1111/j.1753-4887.2001.tb07002.x.

Patterson, R. N. (2003) 'Iron deficiency anaemia: are the British Society of Gastroenterology guidelines being adhered to?', Postgraduate Medical Journal, pp. 
226-228. doi: 10.1136/pmj.79.930.226.

Pietra, V., Degrassi, S. and Fusi-Schmidhauser, T. (2020) 'The Dark Side of Iron', The American journal of medicine, 133(1), pp. e7-e8.

Rimon, E. et al. (2005) 'Are we giving too much iron? Low-dose iron therapy is effective in octogenarians', The American journal of medicine, 118(10), pp. 11421147.

Smith, D. L. (2000) 'Anemia in the elderly', American family physician, 62(7), pp. 1565-1572.

Smith, M. A. et al. (1997) 'Iron accumulation in Alzheimer disease is a source of redox-generated free radicals', Proceedings of the National Academy of Sciences of the United States of America, 94(18), pp. 9866-9868.

de Sousa, M. and Porto, G. (1998) 'The immunological system in hemochromatosis', Journal of hepatology, 28 Suppl 1, pp. 1-7.

Tripathi, K. D. (2008) 'Haematinics and Erythropoietin', Essentials of Medical Pharmacology, pp. 579-579. doi: 10.5005/jp/books/10282_45.

Tripathi, K. D. (2013a) 'Chapter-06 Adverse Drug Effects', Essentials of Medical Pharmacology, pp. 82-98. doi: 10.5005/jp/books/12256_6.

Tripathi, K. D. (2013b) 'Chapter-43 Haematinics and Erythropoietin', Essentials of Medical Pharmacology, pp. 599-612. doi: 10.5005/jp/books/12021_47.

Valk, B. de, de Valk, B. and Marx, J. J. M. (1999) 'Iron, Atherosclerosis, and Ischemic Heart Disease', Archives of Internal Medicine, p. 1542. doi: 10.1001/ archinte.159.14.1542.

Vani, S. and Grover, A. (2003) 'Effects of Maternal Iron Deficiency Anemia and Iron Supplementation on Fetus', Textbook of Neonatal Hematology-Oncology, pp. 24-24. doi: 10.5005/jp/books/10925_4.

Wolf, M. et al. (2020) 'Effects of Iron Isomaltoside vs Ferric Carboxymaltose on Hypophosphatemia in IronDeficiency Anemia: Two Randomized Clinical Trials', JAMA: the journal of the American Medical Association, 323(5), pp. 432-443. 